\title{
El modelo de difusión de innovaciones de Rogers en la bibliometría mexicana
}

The diffusion of innovations model of Rogers in the mexican bibliometrics

Rubén Urbizagástegui-Alvarado

Universidad de California en Riverside, Estados Unidos

ruben@ucr.edu

\section{ReSUMEN:}

El objetivo de este artículo es explorar el modelo de difusión de las innovaciones propuesto por Rogers aplicándolo a los productores de la literatura publicada sobre las "metrías" en México. Como unidades de análisis fueron tomados cada uno de los artículos publicados en revistas académicas, capítulos de libros, y trabajos presentados en congresos que trataron algunos de los aspectos de los estudios métricos o las aplicaciones de estas técnicas en una disciplina o subcampo determinado en México, o por mexicanos que publicaron documentos sobre este asunto fuera de México. El periodo cubierto va desde los primeros trabajos publicados a inicios de la década de los 70 hasta diciembre del 2017. Se encontraron 690 documentos producidos y publicados por 1410 autores diferentes en el periodo de investigación. La teoría de la difusión de las innovaciones ayuda a acompañar el desarrollo histórico de la bibliometría mexicana identificando adecuadamente a sus innovadores, adoptantes iniciales, y demás categorías de la teoría de la difusión de las innovaciones de Rogers.

Palabras clave: Difusión de innovaciones, Bibliometría, Cienciometría, México.

\section{ABSTRACT:}

The objective of this article is to explore the model of diffusion of the innovations suggested by Rogers applying it to the producers of the literature published on the "metrics" in Mexico. As units of analysis were taken each of the articles published in academic journals, book chapters, and papers presented at conferences that addressed some of the aspects of metric studies or the applications of these techniques in a discipline or subfield in Mexico or by Mexicans who published documents on this matter outside of Mexico. The period covered ranges from the first papers published at the beginning of the 1970s up to December 2017.690 documents were produced and published by 1410 different authors in the research period. The theory of the diffusion of innovations helps to accompany the historical development of Mexican bibliometrics by adequately identifying its innovators, initial adopters, and other categories of the theory of the diffusion of innovations of Rogers.

KEYWORDS: Bibliometrics, Scietometrics, Difussion of innovations, Mexico.

\section{INTRODUCCIÓN}

Uno de los problemas en la comunicación del conocimiento por medio de la literatura publicada, y poco explorada en el campo de la Bibliotecología y Ciencias de la Información (BCI), es el análisis de la forma en que aparece una "nueva línea de investigación" que no existía antes en el campo científico, luego cómo en esta nueva línea de investigación que se abre a exploraciones los autores comienzan a publicar los primeros artículos o documentos, cómo luego aparecen nuevos seguidores o adoptantes de esa nueva línea de investigación y, poco después, cómo otros seguidores comienzan a publicar sobre el mismo asunto. Este proceso genera posteriormente una acumulación y crecimiento de la literatura publicada, así como una multiplicación de los autores adoptantes de esta nueva línea de investigación. Es decir, el asunto de común interés adoptado se difunde, pues, "los resultados de la investigación no se consideran científicos a menos que se recojan, se difundan, se compartan y, finalmente, se transformen en una propiedad común, al ser publicada formalmente" (Ziman, 2003, p. 115.). La norma en una comunidad científica es que el conocimiento se haga público en otras palabras, que se publique, lo que significa que de alguna manera se aceptaron y se adoptaron

Recepción: 27 de febrero de 2019 | Aceptación: 29 de abril de 2019 | Publicación: 28 de octubre de 2019 
las ideas, técnicas, metodologías o formulaciones teóricas comunicadas a través de los asuntos investigados en cualquier nueva línea de exploración. El proceso de diseminación de los resultados de una investigación, por medio de la literatura publicada es así muy similar al proceso de difusión de las innovaciones.

La difusión de innovaciones se refiere al proceso mediante el cual una innovación es diseminada entre los miembros de un sistema social, por medio de ciertos canales de comunicación (Rogers, 1963). La innovación puede ser una idea nueva, una práctica o un objeto que sea novedoso para los miembros de un sistema social o la población que participa de ese entorno social (Mahajan y Peterson, 1985). Estas mismas características se presentan en la difusión de la literatura científica producida por los investigadores en los diferentes campos del conocimiento. Por lo tanto, la difusión de la literatura científica puede ser estudiada también como la difusión de una innovación. La literatura científica no deja de ser la expresión de una innovación especialmente cuando estas exploran nuevas ideas, nuevos métodos, nuevas líneas de investigación no existentes en periodos anteriores.

En el campo de la bibliotecología y ciencia de la información, donde se incorporaron las investigaciones sobre bibliometría, cienciometría y otras "metrías", las innovaciones en la forma de nuevas ideas se diseminan entre los miembros del campo, a través de las revistas académicas, los congresos, workshops especializados, mesas redondas y eventos similares que conforman los diversos tipos de canales de comunicación, sin olvidar por supuesto los cursos que se ofrecen en los distintos programas de formación académica y donde los profesores juegan un papel importante en la difusión del conocimiento. De lo expresado anteriormente se puede construir la pregunta de investigación de la siguiente manera:

¿La diseminación de la literatura publicada sobre las “metrías" en México sigue el modelo de difusión de innovaciones propuesto por Rogers?

El objetivo de este artículo es explorar el modelo de difusión de las innovaciones propuesto por Rogers (1963) aplicándolo a los productores de la literatura publicada sobre las "metrías" en México. Para alcanzar el objetivo propuesto, este artículo está organizado de la siguiente manera: después de una somera introducción y establecimiento del objetivo del artículo, se ofrece un marco teórico de la teoría de la difusión de innovaciones y una revisión de la literatura pertinente. Luego se establece la metodología y se explican los procedimientos ajustados a sus objetivos, se describen los resultados y las conclusiones. Finalmente se ofrece una lista de la literatura consultada en el proceso de elaboración de este trabajo.

\section{Marco TeÓRico y ReVISIÓN DE LA LITERATURA}

Las primeras investigaciones sobre la teoría de la difusión de las innovaciones aparecieron a finales del siglo XIX, cuando en Europa surgen la sociología y la antropología como nuevos campos del conocimiento en las ciencias sociales. El francés Gabriel Tarde (1890, 2003; 2010), es considerado uno de los abanderados de la difusión de las innovaciones al proponer su "teoría de la imitación" basada en tres conceptos fundamentales: invención, imitación y oposición. Tarde afirmaba que cuanto más similares son las innovaciones y las ideas que ya son aceptadas, mayor será la probabilidad de que las innovaciones sean adoptadas. De esa manera la adopción seguiría una curva en forma de $S$ en relación con el tiempo. Su teoría sobre la adopción de las innovaciones no fue seguida por estudios empíricos en esta área, llevando casi 50 años para ser aplicado en un estudio sobre semillas híbridas de maíz en los Estados Unidos (Rogers, 2003).

La teoría de la difusión de las innovaciones propuesta por Rogers (2003) busca explicar la forma en que las innovaciones son adoptadas por una población. La tasa de conocimiento de una innovación por la población adoptante (por ejemplo, los modelos bibliométricos) siempre será mayor que la tasa de adopción de la innovación, la decisión de adoptar una innovación es un hecho individual que ocurre durante un largo período y que consiste de una serie de acciones diferenciadas que comprende: 
a) Conocimiento: ocurre cuando un individuo es expuesto a la innovación y toma conocimiento de cómo opera esta innovación.

b) Persuasión: ocurre cuando un individuo, que ya fue expuesto a la innovación, adopta una actitud favorable o desfavorable con relación a la innovación.

c) Decisión: ocurre luego de la persuasión, cuando el individuo desarrolla acciones que lo llevan a adoptar o rechazar una innovación.

d) Implementación: ocurre cuando el individuo decide adoptar la innovación y lo pone en práctica, es decir, comienza a utilizarlo.

e) Confirmación: ocurre cuando el individuo busca reforzar la decisión de implementación adoptada, pudiendo alterar su decisión si está expuesto a mensajes conflictivos sobre la innovación.

Una población de posibles adoptantes puede dividirse en cinco diferentes segmentos, basados en su propensión a adoptar una innovación específica. Cada segmento mantiene una actitud propia frente a la adopción de las innovaciones, pues una innovación se propaga solamente cuando está lo suficientemente "madura" como para satisfacer las necesidades innovadoras de cada segmento. Siguiendo las propuestas de Rogers (2003) estos segmentos están compuestos por:

i. Losinnovadores: el proceso de difusión comienza con un pequeño grupo de innovadores, visionarios, imaginativos, y creativos. Promotores del cambio que buscan y promueven la innovación, pues, a menudo gastan su tiempo, energía y creatividad en el desarrollo y adopción de nuevas ideas, nuevos métodos, nuevas formas de enfocar los problemas. Les gusta hablar sobre sus hallazgos, pero no son los mejores difusores de las propias innovaciones que generan. Por lo general son menos del $2.5 \% \mathrm{de}$ la comunidad estudiada.

ii. Los adoptantes iniciales : por lo general son líderes de opinión que prueban las nuevas ideas, las nuevas formas de enfocar los problemas, pero lo hacen de una manera muy cuidadosa. Evalúan objetivamente si la innovación va a dar como resultado beneficios objetivos y cuando están convencidos de estos resultados ventajosos tienen una alta probabilidad de adoptar la innovación. Una vez que las ventajas comienzan a ser vistas, estos individuos empiezan las adopciones iniciales. Los adoptantes iniciales tienden a ser más exitosos, con más contactos y mejor informados, lo que los lleva a ser más respetados en la comunidad en que actúan. Aparentemente, les encanta hablar de sus éxitos, haciendo que la fama de una innovación se intensifique después de pasar por este segmento. Si los comentarios de este grupo sobre la innovación son positivos, tiende a producirse el despegue del proceso de difusión. Al actuar con mayor objetividad pueden definir mejores estrategias de difusión e implementación de la innovación. Por lo general este segmento está formado por $13.5 \%$ de los individuos de la comunidad estudiada.

iii. La mayoría temprana: los integrantes de este grupo por lo general son pragmáticos y cómodos con innovaciones moderadamente progresivas, pero no actúan sin una prueba sólida de los beneficios de la innovación. Necesitan sentir la seguridad difundida por los líderes de opinión. Son seguidores influenciados por las corrientes principales, pero muy cautelosos con las modas. Sienten temor por el riesgo, y desaprueban la complejidad. Están buscando soluciones simples y probadas para hacer lo que ya saben hacer. Para lograr que este grupo adopte la innovación se necesita desarrollar estrategias de difusión diferentes: el uso de presentaciones, comunicaciones, informes de resultados formales o informales. Cuando este grupo se da cuenta de que la innovación será adoptada de todas maneras por la comunidad en la que actúan, buscan mayores informaciones y verifican los beneficios que lograron los que ya hacen uso de la innovación y no desean quedarse sin los beneficios de la adopción de la innovación, pero en esta etapa lo nuevo deja de ser una novedad para comenzar a convertirse en algo normal. Por lo general este segmento está formado por $34 \%$ de los individuos de la comunidad estudiada. 
iv. La mayoría tardía : son pragmáticos conservadores que tienen miedo del riesgo y se sienten incómodos con las nuevas ideas. Su único estímulo para adoptar la innovación es el miedo a quedarse desfasados y no tienen más opción que cambiar. Por lo tanto, sólo van a seguir los patrones ya establecidos. Son conservadores ortodoxos. Por lo general este segmento está formado por $34 \%$ de los individuos de la comunidad estudiada.

v. Los rezagados : los integrantes de este grupo por lo general son personas que ven como un alto riesgo el adoptar las innovaciones. Siempre buscan argumentos para rebatir las innovaciones. No necesariamente porque sienten que están equivocados, sino porque las innovaciones son tan diferentes a lo que ya conocen que acaban desafiando sus paradigmas. Por lo general este segmento está formado por $16 \%$ de los individuos de la comunidad estudiada.

La Figura 1, muestra la clasificación de los grupos de posibles adoptantes dividido en cinco segmentos diferentes distribuidos en la forma de una curva de distribución normal, a la que se le conoce también como una distribución de Gauss.

Figura 1. Distribución de los adoptantes

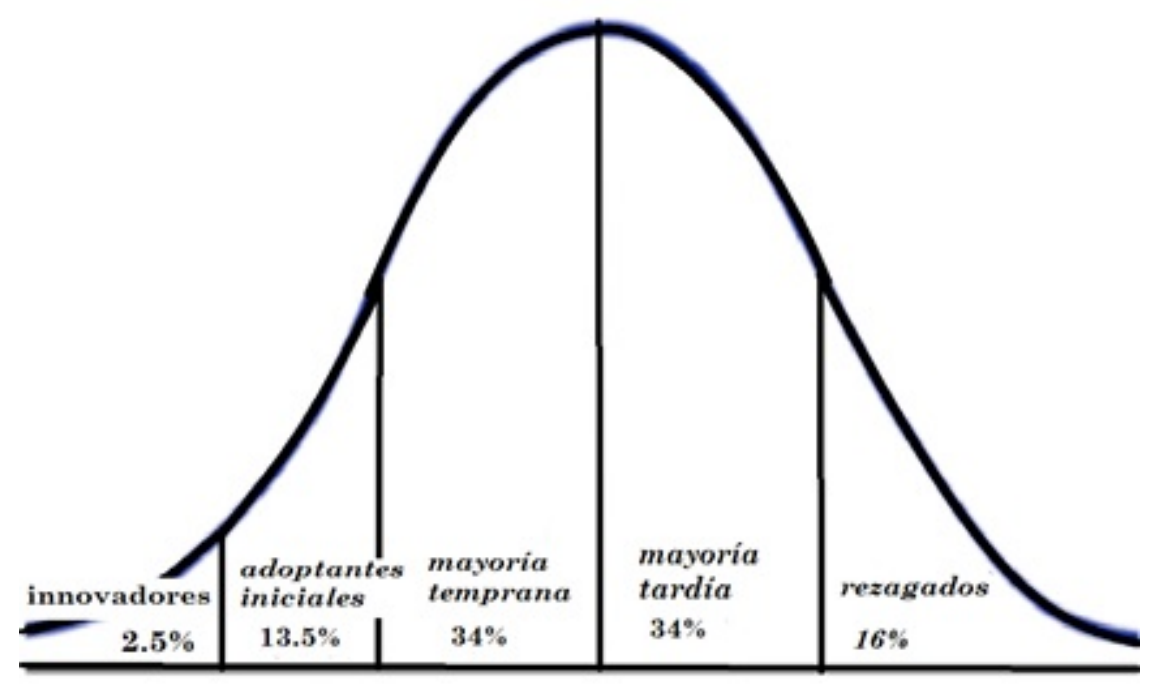

Fuente: Adaptado de Rogers, 2003.

Sin embargo, la adopción de una nueva idea es difícil, aun si las innovaciones muestran ventajas inmediatamente visibles. Las innovaciones en las ideas y en las técnicas necesitan un largo periodo de tiempo para ganar aceptación por los agentes adoptantes. Desde el momento en que estas nuevas ideas son conocidas hasta que son efectivamente adoptadas existe un largo recorrido, pues, son susceptibles a las subjetividades de los agentes y al intercambio de información entre los adoptantes. La difusión de la innovación es un proceso social antes que una simple cuestión técnica. En esta etapa del proceso los medios de comunicación de masas y las conexiones interpersonales juegan un papel importante. Los canales de comunicación de masas son más efectivos para promover el inicio del proceso de difusión, ya que tienen la capacidad de llegar a muchas personas más rápidamente. Sin embargo, la comunicación de masas es poco efectiva en reforzar y confirmar las bondades de una innovación; en esta etapa los canales de comunicación interpersonales son más efectivos para los efectos de persuasión.

Determinada la importancia de las comunicaciones interpersonales durante el proceso de difusión, se afirma que los medios de masas son importantes en el proceso de difusión de la información sobre las nuevas ideas, pero que el intercambio de información entre los agentes es esencial para garantizar la adopción. Se sabe que el éxito de la difusión de una nueva idea es el resultado del intercambio de información a través de redes interpersonales. Si el primer adoptante de una innovación discute esto con otros dos miembros de un sistema, 
y cada uno de ellos con otros dos y estos cuatro con otros cuatro y así sucesivamente, finalmente se tendrá una difusión y expansión exponencial. Por lo tanto, al trazar la distribución del número de agentes que adoptaron las nuevas ideas o innovaciones de forma acumulada en relación con el tiempo, resultará en una distribución sigmoidal, conocida también como "curva $S$ ", como se ilustra en la Figura 2. Es en esta curva sigmoidal en forma de $S$ invertida que se intentará encontrar la difusión de las ideas sobre los estudios "métricos" de la información en México.

Figura 2. Curva $S$ típica de un proceso de adopción

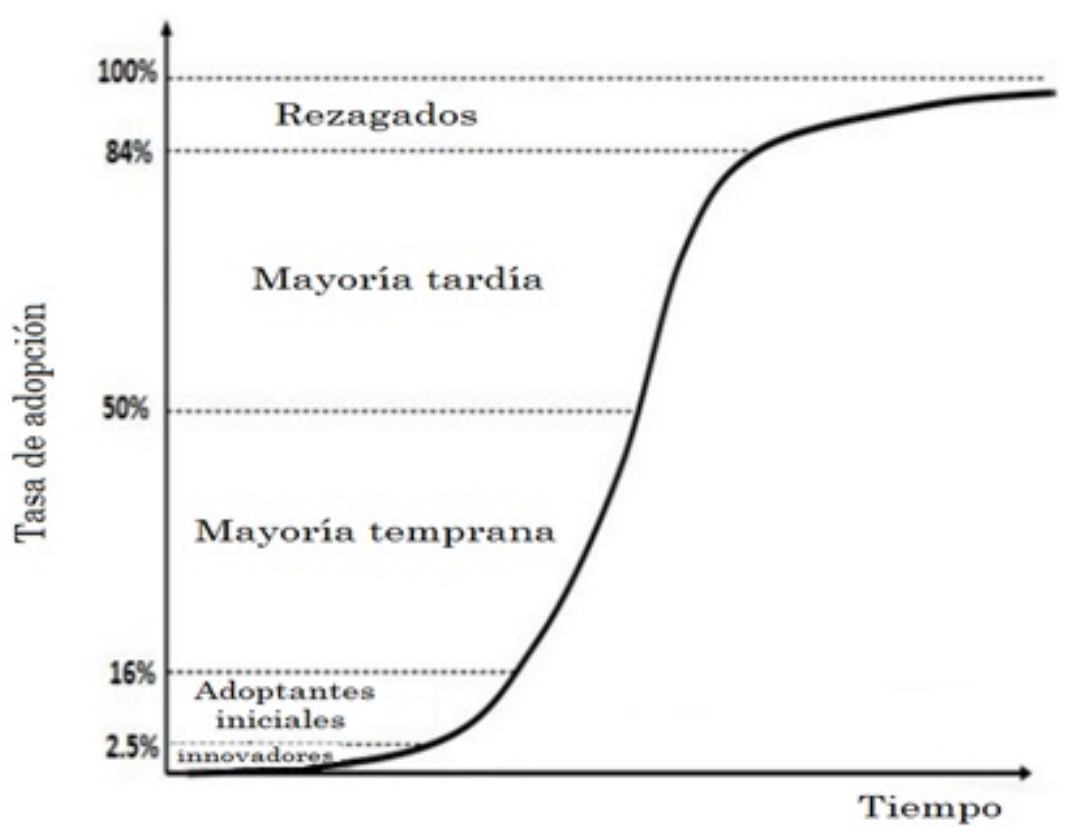

Fuente: Elaborado por el autor

Fuentes Navarro (2005) analiza la influencia del enfoque de la difusión de innovaciones en la investigación latinoamericana de la comunicación, especialmente en la década de los años sesenta, pero la teoría de la difusión de la innovación se ha aplicado en diferentes campos para comprender cómo las personas transfieren nuevas ideas, nuevas habilidades en el tratamiento y conocimiento de enfermedades o estrategias educativas; por ejemplo, en el área de diabetes (Lien y Jiang, 2017), la salud pública (Balas y Chapman, 2018); el análisis de por qué se utilizan los libros electrónicos académicos y cómo influir en el comportamiento de los usuarios de las bibliotecas académicas para aumentar el uso de los libros electrónicos (Raynard, 2017); la comunicación de innovaciones entre mujeres agricultoras en Tanzania (Isaya, Agunga y Sanga, 2018); el uso, citación y difusión de tres herramientas de software de cartografía bibliométrica (CiteSpace, HistCite y VOSviewer) en artículos científicos (Pan, Yan, Cui y Hua, 2018); y Oorschot, Hofman y Halman (2018) realizaron una revisión sistemática y exhaustiva de la literatura sobre adopción de innovaciones, que incluyen 1260 artículos, para identificar los pilares teóricos y las tendencias de las investigaciones en adopción de la innovación.

No está demás afirmar que la bibliometría mexicana ha estado siendo explorada por Restrepo Arango y Urbizagástegui-Alvarado (2016) quienes analizaron el idioma, la tipología documental, el lugar de publicación, las revistas más frecuentemente seleccionadas para publicar los artículos, las áreas del conocimiento donde se publican estos documentos, la colaboración y el crecimiento de las publicaciones sobre bibliometría, cienciometría, informetría, etc., producida por autores mexicanos y/o extranjeros en México, así como por mexicanos en el extranjero desde 1971 hasta 2012. Realizaron también un análisis de las co-palabras utilizando las palabras clave presentes en los documentos publicados sobre las metrías en México (Restrepo Arango y Urbizagástegui Alvarado, 2017); finalmente analizaron la red de coautorías existentes en las publicaciones sobre las metrías en México (Urbizagástegui-Alvarado y Restrepo Arango, 2018). Sin 
embargo, en el campo de la bibliometría y/o la bibliotecología y ciencias de la información, el modelo de difusión de innovaciones de Rogers aún no ha sido explorado.

\section{Material y métodos}

Como unidades de análisis fueron tomados cada uno de los artículos publicados en revistas académicas, capítulos de libros, y trabajos presentados en congresos que trataron algunos de los aspectos de los estudios métricos (bibliometría, cienciometría, informetría, etc.) o las aplicaciones de estas técnicas en una disciplina o subcampo determinado en México o por mexicanos que publicaron documentos sobre este asunto fuera de México. Se excluyeron los autores de libros, tesis, monografías y literatura gris por dos razones. Primero, porque los libros comienzan como artículos publicados en revistas especializadas; y segundo, porque las tesis, monografías y literatura gris no son indexadas en muchas de las bases de datos bibliográficas consultadas para esta investigación. El periodo cubierto tiene en cuenta los primeros trabajos publicados en la década de los 70 hasta diciembre del 2017.

Para recolectar los datos fueron efectuadas búsquedas usando los términos listados en el Anexo A en sus diferentes acepciones idiomáticas (inglés, francés, alemán, portugués, español, etc.) y en múltiples combinaciones booleanas en los títulos, palabras clave y resúmenes de las bases de datos bibliográficas y portales de bibliotecas listadas en el Anexo B. Las referencias identificadas fueron después exportadas a EndNote X8 para la elaboración de una base de datos sobre el asunto. Posteriormente fue realizada una minuciosa lectura de cada uno de los documentos identificados en la búsqueda, dedicando especial atención a cada referencia bibliográfica listada en el documento leído. Después cada referencia bibliográfica relativa al asunto de investigación era confrontada con la base de datos e incorporada si no había sido identificada en la búsqueda anterior. Las referencias duplicadas fueron eliminadas, manteniéndose solamente una única referencia no repetida. La identificación de la pertinencia del documento fue una tarea ardua y difícil, porque con mucha frecuencia las palabras clave fueron insuficientes y a veces falsos indicativos. Para identificar a los autores mexicanos se analizó la afiliación institucional de cada autor identificado en cada referencia recuperada. Muchas veces fue necesario consultar la Plataforma del Sistema Nacional de Investigadores de México para realizar esta verificación o buscar los "currículo vitae" de los autores, y en no pocas situaciones, se realizó minería de textos. La nacionalidad y la afiliación institucional fueron también indagadas por medio de mensajes personales enviados vía el correo electrónico al investigador identificado. Con toda esa estrategia y la lectura minuciosa de muchos de los artículos identificados fue producida la base de datos (que sirve de base al objeto de esta investigación) que contiene referencias de artículos de revistas, capítulos de libros, comunicaciones presentadas en congresos, una que otra nota editorial y cartas enviadas a los editores de las revistas académicas. Esta base de datos especializada ha estado en construcción permanente por un periodo de más de cinco años.

Para medir los datos de esta literatura se utilizó el modelo de difusión de las innovaciones que se origina de una hipótesis comportamental, que supone que, durante el proceso de difusión de un nuevo producto, este es comunicado a través de ciertos canales de comunicación entre los miembros que comparten un sistema social. En este proceso de comunicación los mensajes están relacionados con las nuevas ideas que los participantes crean y comparten para lograr un entendimiento mutuo. Para medir las categorías de adoptantes se siguió el siguiente proceso propuesto por Rogers (2003):

a) Los innovadores: son al menos $2.5 \%$ de la comunidad estudiada.

b) Los adoptantes iniciales: este segmento está formado por $13.5 \%$ de los individuos de la comunidad estudiada.

c) La mayoría temprana: está formado por $34 \%$ de los individuos de la comunidad estudiada. 
d) Mayoría tardía: por lo general este segmento está formado por $34 \%$ de los individuos de la comunidad estudiada.

e) Rezagados: este segmento está formado por $16 \%$ de los individuos de la comunidad estudiada.

La suma acumulada de estos segmentos forma el $100 \%$ de la población estudiada $(2.5+13.5+34+34+$ $16=100$ ). Igualmente, el trazo de los valores acumulados forma una curva sigmoidal en forma de $S$ (ver la Figura 2). ${ }^{1}$

\section{Resultados}

La Tabla 1 muestra los tipos de documentos publicados, según los idiomas de publicación. Se identificaron 690 documentos publicados por 1410 autores diferentes hasta diciembre de 2017. Los autores productores de literatura sobre las "metrías" en México tienen preferencia por presentar sus contribuciones en la forma de artículos publicados en revistas académicas: $78 \%$ de las contribuciones tienen esta forma de publicación, de las cuales $60 \%$ de estos artículos son en español y $17 \%$ en inglés. Las publicaciones en la forma de ponencias presentadas en congresos representan la segunda preferencia, tanto que $17 \%$ del total está compuesto por este tipo de publicaciones, de las cuales $13.5 \%$ son publicados en español y $3.3 \%$ en inglés.

En el periodo estudiado la media de documentos publicados fue de 14.7 documentos por año, con un error padrón de 1.9 documentos, un desvío padrón de 15.136 y una varianza igual a 229.095. Estos resultados parecen naturales en términos de la práctica científica en las ciencias, al final de cuentas las revistas (electrónicas o no) continúan siendo el canal de comunicación formal por excelencia para los científicos y donde los artículos presentan los resultados de la investigación corriente o aún como resultados parciales de una investigación en proceso. De allí que las investigaciones sobre este asunto estén en pleno desarrollo.

Tabla 1. Tipos de documentos publicados según los idiomas

Tipos de documentos

Artículos

Ponencias

Capítulos de libros

Nota editorial

Nota periodística

Total

\section{Idiomas}

Español Inglés Francés Portugués Total

$\begin{array}{rcccc}415 & 120 & 1 & 2 & 538 \\ 93 & 23 & 1 & - & 117 \\ 19 & 3 & - & 2 & 24 \\ 5 & - & - & - & 5 \\ 6 & - & - & - & 6 \\ 538 & 146 & 2 & 4 & 690\end{array}$

La Figura 3 muestra la representación gráfica de los tipos de documentos publicados sobre las "metrías" en México hasta diciembre de 2017. La preferencia mayoritaria es por las publicaciones como artículos en revistas arbitradas. 
Figura 3: Tipos de documentos producidos

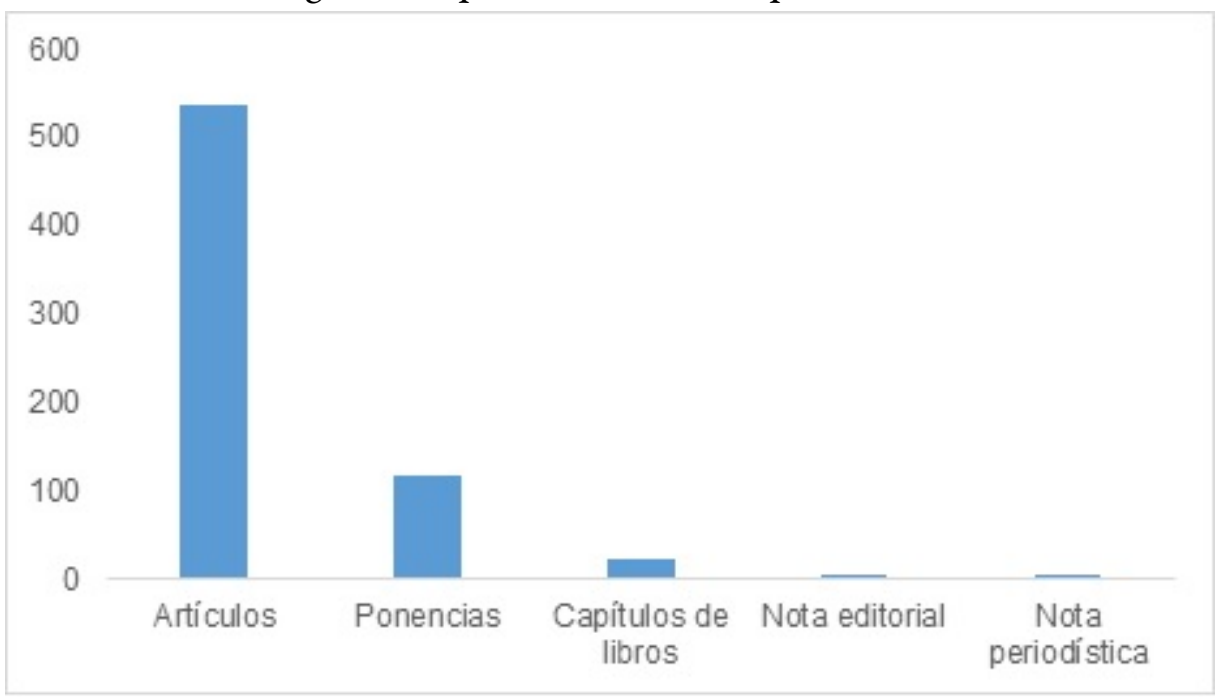

La Tabla 2 muestra el número de autores diferentes que produjeron documentos sobre las "metrías" desde 1971 hasta diciembre de 2017. Se consideró que un autor entró al campo, es decir adoptó la innovación, en el año de la primera publicación de cualquiera de los tipos de documentos señalados y mostrados en la Tabla 1. El primer artículo fue publicado por Jorge Robles Glenn en 1971, cuatro años más tarde Pedro González Blasco publicó otro documento. Después del año 1976, paulatinamente aumenta el número de autores que adoptan esta innovación, con la excepción de los años 1979 y 1982, años en los que ningún nuevo autor adopta la innovación. A partir de 1983 se observa una adopción constante que no para de crecer con muy pocas fluctuaciones hasta diciembre de 2017.

Tabla 2. Número de autores de documentos según los años, 1971-2017

\begin{tabular}{cccccccc} 
Años & $\begin{array}{l}\text { No de } \\
\text { autores }\end{array}$ & Años & $\begin{array}{l}\text { No. de } \\
\text { autores }\end{array}$ & Años & $\begin{array}{l}\text { No. de } \\
\text { autores }\end{array}$ & Años & $\begin{array}{c}\text { No. de } \\
\text { Autores }\end{array}$ \\
\hline 1971 & 1 & 1983 & 3 & 1995 & 19 & 2007 & 60 \\
1972 & 0 & 1984 & 2 & 1996 & 24 & 2008 & 70 \\
1973 & 0 & 1985 & 4 & 1997 & 19 & 2009 & 70 \\
1974 & 0 & 1986 & 6 & 1998 & 24 & 2010 & 109 \\
1975 & 1 & 1987 & 14 & 1999 & 36 & 2011 & 100 \\
1976 & 1 & 1988 & 10 & 2000 & 42 & 2012 & 98 \\
1977 & 3 & 1989 & 8 & 2001 & 31 & 2013 & 87 \\
1978 & 5 & 1990 & 9 & 2002 & 43 & 2014 & 104 \\
1979 & 0 & 1991 & 4 & 2003 & 31 & 2015 & 63 \\
1980 & 1 & 1992 & 10 & 2004 & 38 & 2016 & 48 \\
1981 & 3 & 1993 & 13 & 2005 & 43 & 2017 & 91 \\
1982 & 0 & 1994 & 22 & 2006 & 40 & &
\end{tabular}


La Figura 4 muestra la curva de adopción según el tiempo determinado en años de la bibliometría mexicana. Se observa una progresión temporal desde unos niveles bajos al inicio, pero aun no aparecen indicios de acercamiento a un punto de saturación en el nivel más alto. La transición se produce en una región caracterizada por una fuerte aceleración intermedia que permite describir esta evolución, que finalmente se desacelera y eventualmente crece muy poco. Su gráfica busca una típica forma de $S$ que en este caso de la bibliometría mexicana aun no aparece claramente. Esta forma de curva cóncava al final de la figura indicaría el aparecimiento de un proceso de saturación, pero esta figura muestra una progresión continua que significa que la bibliometría mexicana está en plena expansión. En otras palabras, más o menos hasta 1986, los modelos bibliométricos están libremente disponibles, pero pocos lo adoptan.

Cuando la bibliometría mexicana se encuentra en esta fase inicial es apreciada por su nivel de novedad y causa entre los interesados el entusiasmo suficiente como para que deseen adoptarlo antes que los demás, aun sin conocer a fondo sus características y modelos matemáticos. A partir de esa fecha hay un rápido crecimiento inicial y tardío. En esta etapa la bibliometría se encuentra en pleno desarrollo. En esta fase es donde se presenta una apertura en dirección a su formalización; posiblemente se dicten cursos de introducción a la bibliometría y se introduzcan cursos especializados en las escuelas de formación académica y aparecen los nuevos competidores, nuevos agentes entran al campo de la producción académica sobre bibliometría. La bibliometría goza de un posicionamiento privilegiado en el campo de la BCI. Todos los esfuerzos de los agentes en competencia están encaminados a conservarla o aumentarla, con el fin de no ceder terreno ante los competidores que cada vez son más numerosos. En la etapa de crecimiento tardío la bibliometría mexicana se encuentra en plena maduración de su ciclo de vida. Se consolida la competencia, la producción académica es rentable y la calidad del producto es un factor diferenciador.

Figura 4. Curva de adopción

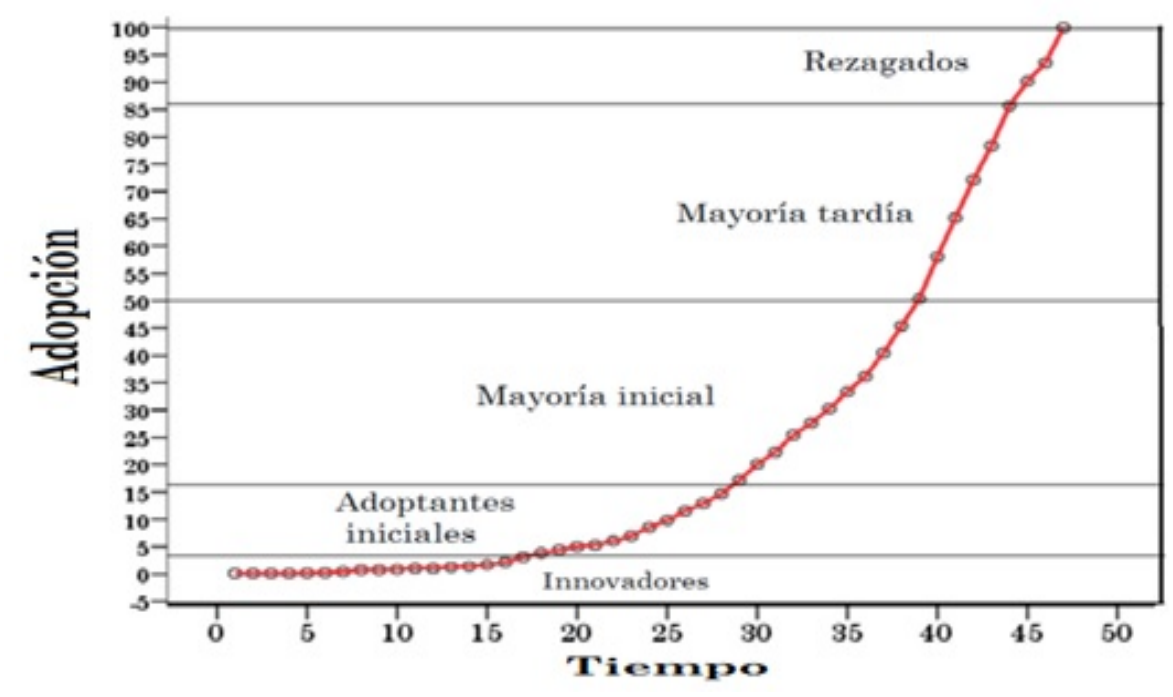

Finalmente se entra a un proceso de saturación y comienza la obsolescencia, lo que propicia la creación de un nuevo paradigma y por ende una nueva oportunidad de renovación o extinción. El asunto entra a una etapa de envejecimiento y declive, pero en el caso de la bibliometría mexicana este punto aun no aparece claramente en la figura sigmoidal, significando que está en pleno desarrollo y expansión (ver Figura 4).

El grupo de innovadores está conformado por 30 autores (2.13\% de la población total) y les llevó 16 años en sentar las bases de la adopción del estudio de las "metrías" en México. Estos innovadores ${ }^{2}$ son los que aparecen listados en la Tabla 3. 
Tabla 3. Autores innovadores de la bibliometría mexicana

\begin{tabular}{ll} 
Autores innovadores & Autores innovadores \\
\hline Robles Glenn, Jorge & Negrete Gutiérrez, Ma. del Carmen \\
González Blasco, Pedro & Russell Barnard, Jane Margaret \\
Perales de Mercado, Alicia & Perales Ojeda, Alicia \\
López Roblero, Edgar Leonel & Guzmán, Jesús Carlos \\
Pontigo Martínez, Jaime & Oliver Villalpando, Estela \\
Quijano Solís, Álvaro & Jiménez, J. \\
Buttenklepper, Alfredo & Navarro, M. A. \\
Maffey, Lourdes & Rees, Martha W. \\
Delgado, Héctor & Lomnitz, Larissa Adler \\
Ferreira, Delia Valerio & Cameo, León \\
Magaloni de Bustamante, Ana María & Mirande, A. \\
Gorbea Portal, Salvador & Galina, C. S. \\
Sánchez Ambriz, Gerardo & Navarro Fierro, R. \\
Baeza Zavala, B. Leticia & Morales Campos, Estela \\
García Salgado, Ignacio N. & Correa, M. S.
\end{tabular}

El grupo de los adoptantes iniciales está conformado por 15.03\% de la población total que agrupa a 212 autores. Estos autores se demoraron hasta 13 años en adoptar la innovación sobre los diversos aspectos estudiados por las metrías en México. El grupo de la mayoría inicial está conformado por $33.2 \%$ de la población total, que comprende a 468 autores diferentes. Estos autores se demoraron hasta 10 años en adoptar la innovación sobre los diversos aspectos estudiados por las metrías en México. El grupo de la mayoría tardía está conformado por 35.3\% de la población total, y comprende a 498 autores diferentes. Estos autores se demoraron hasta 5 años en adoptar la innovación sobre los diversos aspectos estudiados por las metrías en México. Finalmente, el grupo de los rezagados está conformado por $14.3 \%$ de la población total, y comprende a 202 autores diferentes. Estos autores se demoraron hasta 3 años en adoptar la innovación sobre los diversos aspectos estudiados por las metrías en México. ${ }^{3}$ Este segmento aún está en crecimiento.

\section{Conclusiones}

Este artículo se propuso analizar la difusión de la literatura sobre las "metrías" (bibliometría, cienciometría, infometría, webmetría, archivometría, tecnometría, patentometría, etc.) producida y publicada en México y por mexicanos en el exterior, desde la década de los años 70 hasta diciembre de 2017. Se encontraron 690 documentos producidos y publicados por 1410 autores diferentes en el periodo de investigación. Estos autores prefieren comunicar los resultados de sus investigaciones a través de revistas académicas y comunicaciones en congresos, escritos en el lenguaje nativo que es el español, con poca presencia del inglés y una prácticamente insignificante presencia de otros idiomas.

La teoría de la difusión de las innovaciones propuesta por Rogers $(1963,2003)$ ayuda a acompañar el desarrollo histórico de la bibliometría mexicana identificando adecuadamente a sus innovadores, adoptantes iniciales, y demás categorías de la teoría de la difusión de las innovaciones. El modelo de Rogers propone 2.5\% 
de la población estudiada para los innovadores, en la bibliometría mexicana se identificó un valor cercano a ese porcentaje (2.15\% para los innovadores). El modelo propone $13.5 \%$ de la población para los adoptantes iniciales (se identificó $15.03 \%$ de los autores para esta categoría). El modelo propone 34\% de la población para la mayoría inicial (se identificó $33.2 \%$ de adoptantes para esta categoría). Para la mayoría tardía, el modelo propone $34 \%$ de la población (se identificó 35.3\% de los autores para esta categoría). Finalmente, para los rezagados, el modelo propone $16 \%$ de la población (se identificó $14.3 \%$ de los autores para esta categoría).

En conclusión, con muy pequeñas diferencias no significativas el modelo estima adecuadamente las categorías de adoptantes en la difusión de la bibliometría mexicana. Cuando se escriba la historia de la bibliometría mexicana estos innovadores deberían recibir el reconocimiento que se merecen. Sin embargo, se recomienda mayores investigaciones relacionadas con el modelo de Rogers -un modelo al que no se le ha prestado la debida atención a pesar de que existe desde la década de los sesenta- en el campo de la bibliotecología y ciencia de la información Latinoamericana para validar y generalizar estos resultados.

\section{REFERENCIAS}

Balas, E. A., y Chapman, W. W. (2018). Road map for diffusion of innovation in health care. Health Affairs, 37(2), 198-204.

Fuentes Navarro, R. (2005). Everett M. Rogers (1931-2004) y la investigación Latinoamericana de la comunicación. Comunicación y Sociedad, Nueva época, 4, 93-125.

Isaya, E. L., Agunga, R. y Sanga, C. A (2018). Sources of agricultural information for women farmers in Tanzania. Information Development, 34(1), 77-89.

Lien, A. Shin\#Yu, y Jiang, Yi\#Der (2017). Integration of diffusion of innovation theory into diabetes care. Journal of diabetes investigation, 8(3), 259-260.

Mahajan, V., y Peterson, R. A. (1985). Model for Innovation Diffusion. Beverly Hills, CA: Sage Publications.

Oorschot, J. V., Hofman, E., y Halman, J. (2018). A bibliometric review of the innovation adoption literature. Technological Forecasting and Social Change, 134, 1-21.

Pan, X., Yan, E., Cui, M., y Hua, W. (2018). Examining the usage, citation, and diffusion patterns of bibliometric mapping software: a comparative study of three tools. Journal of Informetrics, 12(2), 481-493.

Raynard, M. (2017). Understanding academic e-books through the diffusion of innovations theory as a basis for developing effective marketing and educational strategies. The Journal of Academic Librarianship, 43(1), 82-86.

Restrepo-Arango, C., y Urbizagástegui-Alvarado, R. (2016). Acercamiento a los estudios Biliométricos, Cienciométricos e Infométricos en México. Informação \& Sociedade: Estudos, 26(1), 51-71.

Restrepo Arango, C., y Urbizagástegui Alvarado, R. (2017). Red de co-palabras en la bibliometría mexicana. Investigación bibliotecológica, 31(73), 17-45.

Rogers, E. M. (1963). Diffusion of innovations. New York: Free Press of Glencoe.

Rogers, E. M. (2003). The Diffusion of Innovations. New York: The Free Press.

Tarde, G. (2003). The Laws of Imitation. New York: Henry, Holt and Co.

Tarde, G. (2010). The Laws of Imitation. Charleston: Bibliolife.

Urbizagástegui-Alvarado, R. y Restrepo Arango, C. (2018). La red de co-autores en la bibliometría Mexicana. Encontros Bibli: revista eletrônica de biblioteconomia e ciência da informação, 23(51), 74-94.

Ziman, J. (2003). ¿Quées la ciencia? Madrid: Cambridge University Press. 


\title{
ANEXO A: Términos de búsqueda
}

\author{
México \\ Índice $\mathrm{h}$ \\ Elitismo \\ Frente de investigación
}

Regla 80/20

Obsolescencia de la literatura

Crecimiento de la literatura

Vida media

Teoría epidémica

Visibilidad

Índice de Pratt

Índice de Price

Ley de Price

Indicadores bibliométricos

Ley de Goffman

Ley de Bradford

Ley de Lotka

Ley de Zipf

Punto de transición

Colegios invisibles

Factor de impacto

Factor de inmediatismo

Análisis de citas

Acoplamiento bibliográfico

Co-citación

Redes sociales

Co-autoría

Colaboración científica

Índice de colaboración

Coeficiente de colaboración

Circulación de la colección

Núcleo básico de periódicos

Indicadores en ciencia y tecnología

Bibliometría

Cienciometría

Informetría

Patentometría

Arquivometría

Bio-bibliometría

Librometría

Tecnometría

Webmetría 


\title{
ANEXO B: Bases de Datos consultadas
}

\author{
INFOBILA de México \\ Humanindex \\ Catálogo de la biblioteca del IIBI/UNAM \\ Periodica \\ Redalyc \\ Scielo México \\ Scielo Brasil \\ Scielo Venezuela \\ Scielo Colombia \\ Scielo Chile \\ Scielo Argentina \\ Scielo Bolivia \\ Library Literature \& Information Science Full Text \\ Library, Information Science \& Technology Abstracts (LISTA) \\ WorldCat \\ HAPI \\ ArticleFirst \\ Science Citation Expanded Index \\ Web of Science \\ Scopus \\ JSTOR \\ Google \\ Google Scholar \\ ISOC \\ ICYT \\ Dialnet \\ Agrícola \\ Biosis \\ CAB Abstracts \\ Medline
}

y otras 120 bases de datos existentes en la Universidad de California en Riverside

\section{Notas}

1 El evaluador B de este artículo afirma, y cito sus palabras, que "el trabajo no lo considero un artículo de investigación” ya que "presenta errores conceptuales". Sería interesante conocer a qué este evaluador considera como un artículo de investigación y que aportase evidencias de los supuestos errores conceptuales. Acusaciones apresuradas más parecen expresiones de iniciantes que carecen de formación académica apropiad

2 El evaluador B afirma que "Los autores que indica como autores innovadores, en su mayoría son autores con una sola aportación. Omite autores importantes como: Judith Licea Ayala, Macías-Chapula, Francisco Collazo, entre otros”. Ser o no ser "importantes" es una cuestión de percepción personal que depende de muchas variables que no están en estudio en este artículo. El modelo de Rogers no está preocupado por esas variables. Este modelo no está preocupado por la identificación de autores "importantes"

3 El evaluador A afirma que "No hay discusión de los resultados. Sin embargo, ...se encuentran otros trabajos que abordan los estudios métricos en México, aunque desde otros enfoques, e incluso la utilización de la teoría de la difusión de la innovación por parte de los EMI para analizar otros campos del conocimiento o especializadades". Y el evaluador 
A ofrece referencias de artículos que usan modelos diferentes y con objetivos diferentes al usado en este artículo. Comparar modelos diferentes no tiene sentido, sería como comparar papas con tomates. Por lo tanto, una discusion de sus resultados tampoco tiene sentido. Se discuten resultados de modelos iguales o similares aunque aplicados a campos diferentes. El evaluador A debería ofrecernos al menos una aplicación de este modelo al campo de la Bibliotecología o Ciencia de la Información. Aunque sea una. No habiendo algo similar, este artículo es original. 